\title{
MICOP: Maximal information coefficient- based oscillation prediction to detect biological rhythms in proteomics data
}

\author{
Hitoshi luchi ${ }^{1,2}$, Masahiro Sugimoto ${ }^{2,3^{*}}$ and Masaru Tomita ${ }^{1,2,4}$
}

\begin{abstract}
Background: Circadian rhythms comprise oscillating molecular interactions, the disruption of the homeostasis of which would cause various disorders. To understand this phenomenon systematically, an accurate technique to identify oscillating molecules among omics datasets must be developed; however, this is still impeded by many difficulties, such as experimental noise and attenuated amplitude.

Results: To address these issues, we developed a new algorithm named Maximal Information Coefficient-based Oscillation Prediction (MICOP), a sine curve-matching method. The performance of MICOP in labeling oscillation or non-oscillation was compared with four reported methods using Mathews correlation coefficient (MCC) values. The numerical experiments were performed with time-series data with (1) mimicking of molecular oscillation decay, (2) high noise and low sampling frequency and (3) one-cycle data. The first experiment revealed that MICOP could accurately identify the rhythmicity of decaying molecular oscillation (MCC $>0.7)$. The second experiment revealed that MICOP was robust against high-level noise (MCC > 0.8) even upon the use of low-sampling-frequency data. The third experiment revealed that MICOP could accurately identify the rhythmicity of noisy one-cycle data (MCC $>0.8)$. As an application, we utilized MICOP to analyze time-series proteome data of mouse liver. MICOP identified that novel oscillating candidates numbered 14 and 30 for C57BL/6 and C57BL/6 J, respectively.

Conclusions: In this paper, we presented MICOP, which is an MIC-based algorithm, for predicting periodic patterns in large-scale time-resolved protein expression profiles. The performance test using artificially generated simulation data revealed that the performance of MICOP for decaying data was superior to that of the existing widely used methods. It can reveal novel findings from time-series data and may contribute to biologically significant results. This study suggests that MICOP is an ideal approach for detecting and characterizing oscillations in time-resolved omics data sets.
\end{abstract}

Keywords: Circadian rhythm, Mutual information, Proteomics

\section{Background}

The circadian rhythm, which involves oscillations over a cycle lasting 24-h, plays a critical role in biological systems [1]. Transcriptional negative feedback loops composed of clock genes are a key component of this mechanism [1-3]. These clock genes regulate downstream gene expression, leading to the 24-h cyclic

\footnotetext{
* Correspondence: msugi@sfc.keio.ac.jp

${ }^{2}$ Institute for Advanced Biosciences, Keio University, Tsuruoka 997-0052, Japan

${ }^{3}$ Health Promotion and Preemptive Medicine, Research and Development Center for Minimally Invasive Therapies, Tokyo Medical University, Shinjuku, Tokyo 160-0022, Japan

Full list of author information is available at the end of the article
}

oscillation of various physiological phenomena such as cell division, energy metabolism, blood pressure, and sleep $[4,5]$. Many molecules are involved in these systems, so comprehensive and multilayered approaches are required to clarify the complex systems. Thus, it is crucial to obtain a deep understanding of the circadian rhythm in order to understand biological systems.

The availability of biological time-course data is key to elucidating circadian rhythms, but there are several difficulties in analyzing biological time-series data. In particular, the accumulation of time-series omics data via the technological innovation of mass spectrometry and DNA sequencers has led to the following problems: (1) low sampling frequency and (2) unstable oscillation. The 
first problem is derived from the generally low sampling frequency of omics datasets because comprehensive approaches such as proteomics and transcriptomics are often expensive and laborious. Several omics studies collected time-course data every $2-4 \mathrm{~h}$ per day and estimated periodicity using 12 to 24 points [6-9]. This sampling frequency of omics data was relatively low compared with those for locomotor activity or tissue luminescence, which were provided every minute [10]. The second problem is the unstable oscillation (such as amplitude decay) of time-course experimental values. There are various types of unstable oscillations in the expression pattern of genes and proteins. For example, previous reports assumed unstable oscillations such as cosine with outlier time points, cosine with a linear trend, cosine with an exponential trend, and decaying cosine as possible natural oscillation phenomena $[11,12]$. These unstable oscillations hamper oscillation detection, in particular for amplitude decay, which is often observed in experimental systems and, is caused by degradation of the metabolic activity of cells and degradation of fluorescent protein [13]. Therefore, novel computational analysis that functions over the time course of omics studies with limited sampling points and amplitude decay should be developed.

Many analytical approaches to predict molecules with oscillating levels from time-series data have been developed. These algorithms were classified into time-domain and frequency-domain methods [14]. Typical time-domain methods are based on cosine curve-based pattern matching and their simple algorithm helps biologists to evaluate their analytical results [14]. For example, COSOPT and chi-squared periodogram are algorithms employing curve fitting and autocorrelation, respectively $[15,16]$. Hughes et al. developed a nonparametric approach using rank by the nonparametric Jonckheere-Terpstra (JT) test and obtained the strength of correlation by Kendal's tau test (JTK) [17]. However, they have disadvantages, such as sensitivity to noise and outliers, and being able to detect only cosine wave-like curves; as such, there is a need for a novel algorithm that can overcome these obstacles. Meanwhile, frequency-domain methods based on spectral analysis are strongly noise-resistant and model-independent [14]. Fisher's G-test estimates periodicity by calculating the periodogram of experimental data and calculating the $P$-value using Fisher's G-statistic [18]. Autoregressive spectral (ARS) analysis is an approach combining time-domain and frequency-domain methods, used to identify molecules with rhythmically oscillating levels in large-scale time-resolved profiles by autoregressive spectral analyses $[19,20]$. Similarly, an approach combining autocorrelation and spectral analysis after removing noise from raw data with a digital filter was also proposed [21]; however, frequency-domain methods are limited by the low sampling frequency and short time period in omics experiments, which means that they are often insufficient to predict the periodicity of large-scale omics datasets [22]. Therefore, developed approaches to characterize oscillating molecules in biological data have been used with success and have contributed to our understanding of biological systems; meanwhile, it has been shown that each method sometimes produces inconsistent results because of noise, sampling rate, and waveform [23]. A novel oscillation prediction method compatible with omics experiments, having a low sampling frequency, was required, for which quantitative evaluation of the performance could also be achieved.

This study developed Maximal Information Coefficient (MIC)-based Oscillating Prediction (MICOP) for analyzing time-series omics datasets with high-level noise and possible decay. MICOP offers unsurpassed performance to identify and characterize oscillating molecules in omics datasets.

\section{Methods \\ Datasets}

Time-resolved data from biological samples are generally obtained every $2-6 \mathrm{~h}$ per day [6-9]. Therefore, we simulated time-series data containing 6-24 points for two cycles for a performance test. Half of these artificially simulated data did not feature oscillation, while the other half did. For oscillating data, to mimic experimental data, noise according to the normal distribution (average $=0$, standard deviation $=0-0.6$ ) was added to the sin curve. The decaying time-series datasets were designed so that the value of the peak in the second cycle is one-third of the value of the peak in the first cycle. The nonoscillating data were random numerical data. Proteomics datasets of C57BL/6 J and C57BL/6, which was already normalized, were downloaded from journal websites $[8,9]$. The simulated data released by Wu et al. are included in MetaCycle, as described below [23, 24].

\section{Design}

A conceptual diagram of MICOP is shown in Fig. 1. The MIC belongs to the nonparametric exploration class, and the score indicates the strength of the linear or non-linear association between variables. First, the mutual information for a scatterplot of $\mathrm{X}$ and $\mathrm{Y}$ is calculated as:

$$
I(X ; Y)=\sum_{Y} \sum_{X} p(X, Y) \log _{2} \frac{p(X, Y)}{p(X) p(Y)}
$$

Where $p(X)$ and $p(Y)$ are marginal probability distribution functions of $X$ and $Y$, and $p(X, Y)$ is joint probability distribution function. Then, to compare the values from different grids and to obtain normalized values between 


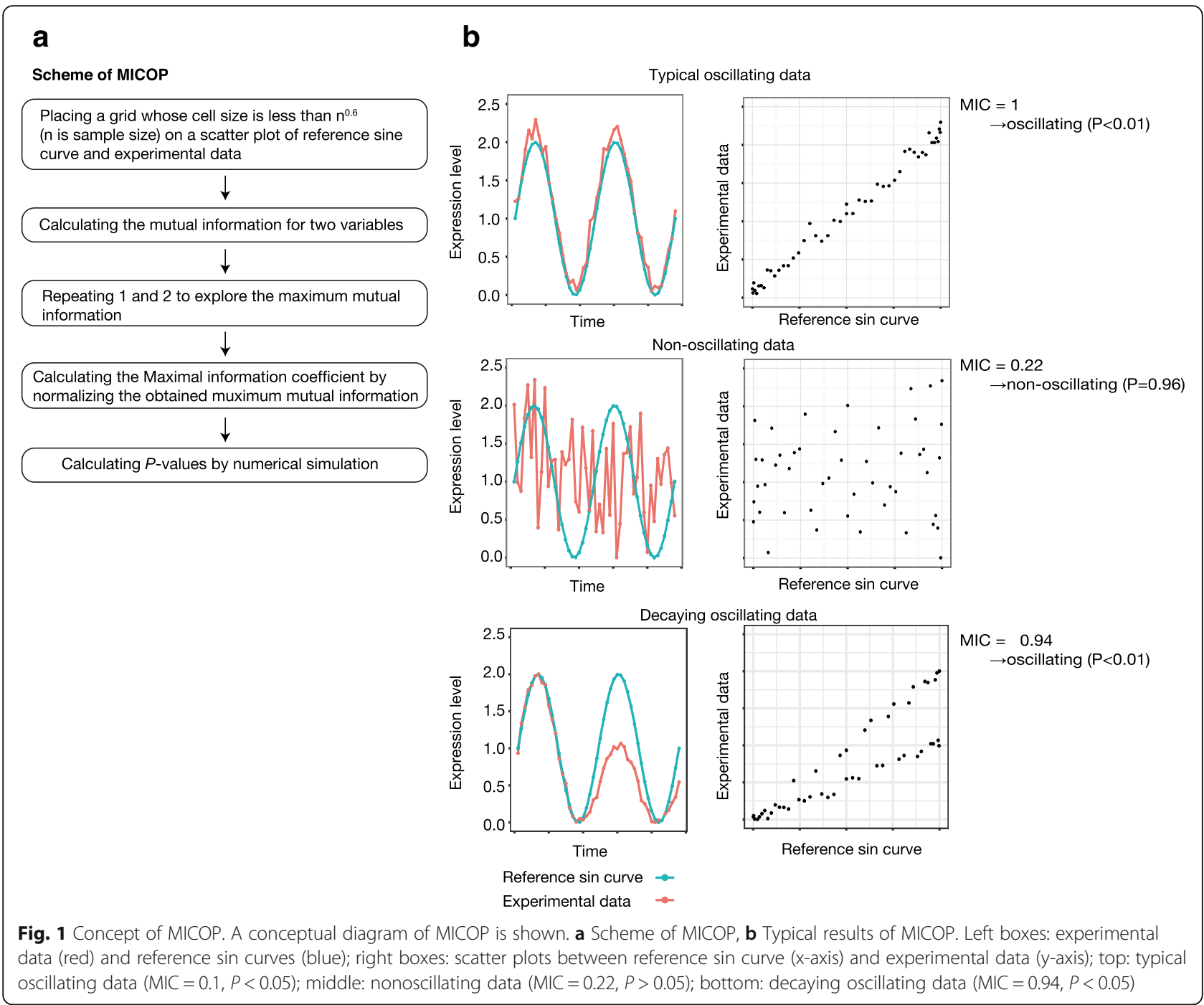

0 and 1, MIC is divided by the lesser number of $\mathrm{X}$ and $\mathrm{Y}$ bins. MIC is calculated as;

$$
M I C(X, Y)=\max _{X, Y<n^{\alpha}} \frac{I(X ; Y)}{\log _{2}(\min (X, Y))}
$$

The algorithm calculates the MIC value between the reference sin curve and experimental data. The same sin curve was used for all input traces. The script for MICOP and its performance test is provided as an $\mathrm{R}$ script. The $P$-values were calculated from the frequency of each MIC value of experimental data and the MIC values that were calculated from the random numbers. The MIC represents the strength of association between the two variables. The MIC between the reference sin curve and targeted data, such as experimental data or simulated data, was calculated using the following steps. Step 1: Grids with different resolutions are introduced to separate the different areas of the scatter plot of the two variables. Step 2: Maximized mutual information at each resolution is selected. Step 3: The mutual information is normalized for each resolution. Step 4: The maximum value among all division methods is MIC. Step 5: to calculate the $P$-value, MIC between the reference curve and 1000 nonoscillating time-series datasets, which comprised random values, was calculated. We compared MIC values and enumerated the occurrences $(k)$ when the MIC score exceeded the score calculated. $k / 1000$ was taken as the $P$-value of the MICOP. Then, we compute the $P$-value as;

$$
P=\frac{1}{1000} \sum_{i=1}^{1000} I(M I C(X p i, Y p i)>M I C(X, Y))
$$

where $I$ is the indicator function, and $\mathrm{X}_{\mathrm{pi}}$ and $\mathrm{Y}_{\mathrm{pi}}$ is the $i$ th permutated version of $\mathrm{X}$ and $\mathrm{Y}$, respectively. If the datasets have missing points, MIC is calculated without the point. 


\section{Performance test}

To test the performance of MICOP, the periodicity of simulated data was determined by MICOP, JTK, ARS, and LS. To compare the precision and sensitivity of MICOP, the MCC was compared [25]. MCC values were calculated as below:

$$
M C C=\frac{\mathrm{TP} \times \mathrm{TN}-\mathrm{FP} \times \mathrm{FN}}{\sqrt{(T P+F P)(T P+F N)(T N+F P)(T N+F N)}}
$$

where TP is the number of true positives, $\mathrm{TN}$ is the number of true negatives, FP is the number of false positives, $\mathrm{FN}$ is the number of false negatives. The false discovery rate is widely used and is calculated from true positive and false positive values. In contrast, MCC is more informative as a value evaluating the performance of the classification method because it is calculated from true positive, false positive, true negative, and false negative values.

\section{Reanalysis of proteomics data}

To verify the practicality of MICOP, we reanalyzed the published time-series data $[8,9,26]$. Briefly, these are proteome datasets of mouse liver sampled every $3 \mathrm{~h}$ for 2 days, and simulated data which are two cycles containing 20 molecules [26]. The MIC and P-value were calculated as described in the Design section.

Programming language, packages, and statistical analysis $\mathrm{R}$ language (ver. 3.3.2) was used for all analyses [27]. Three different random seeds were used; rnorm function was used to generate random numbers according to a normal distribution and runif function was used to generate uniform random numbers. The performance of each method was compared to MICOP by Tukey-Kramer test. The $P$-values were corrected by the Benjamini-Hochberg procedure for multiple testing. A graphical package named ggplot 2 (ver. 2.2.0) was used to draw figures. The Minerva package (ver. 1.4.3) was used to calculate the MIC score, and binning range to calculate MIC score was 0.6 , which is a default value of the $\mathrm{R}$ library. The MetaCycle package (1.1.0) was used for periodicity judgment by ARS, JTK, and LS [21, 23, 24].

\section{Results}

Comparison of MICOP and existing methods for decaying data To test the performance of MICOP, JTK, ARSER, and Lomb-Scargle (LS) for mimicking the decaying time-resolved data, the Matthews correlation coefficient (MCC) values were calculated to differentiate significantly oscillating data from nonoscillating data using time-series simulation data, including 100 sets of oscillating data and 100 sets of nonoscillating ones (Fig. 2, Additional file 1) [17, 20]. Two-way ANOVA with Method and sampling frequency as factors revealed significant effects of Method $(\mathrm{F}=631.8, P<0.005)$, sampling frequency $(\mathrm{F}=810.1, P<0.005)$ and Method $\mathrm{x}$ sampling frequency interaction $(\mathrm{F}=122.9, \quad P<0.005)$. MCC values were $0.72(P<0.005), 0.40(P<0.005), 0.082$ $(P<0.005)$, and $0.00(P<0.005)$ for MICOP, ARS, JTK, and LS, respectively, when the sampling interval was $4 \mathrm{~h}$ (Fig. 2). The MCC values increased as the sampling frequency increased, and these values became almost equal to 1 in all methods at 1 -h interval sampling. The MCC values of MICOP were 0.7 or more at all sampling frequencies and were the highest at a sampling interval of 1-3 $\mathrm{h}$, followed by ARS and JTK. LS did not function as a classifier at a sampling interval of $1-3 \mathrm{~h}$.

\section{Comparison of MICOP and existing methods for noisy or} low-sampling-frequency or one-cycle data

We compared the accuracy of MICOP and existing methods for time-series data containing noise and having a low sampling frequency without attenuation (Fig. 3a and b, Additional file 2). Initially, we quantitatively evaluated the degradation of classification performance due to the noise of MICOP (Fig. 3a). Two-way ANOVA with Method and noise level as factors revealed significant effects of Method $(\mathrm{F}=1099.4, P<0.005)$, noise level $(\mathrm{F}=643.2, P<0.005)$ and method $\mathrm{x}$ noise level interaction $(\mathrm{F}=475.5, P<0.005)$. The $\mathrm{MCC}$ values were 0.8 or more, except for LS, in all conditions, even if the noise was 0.500; however, LS did not function as a classifier when the noise was 0.375 or more.

The performance of MICOP as a classifier for low-sampling-frequency unattenuated data was also quantitatively evaluated (Fig. 3b). Two-way ANOVA with Method and sampling frequency as factors revealed significant effects of Method ( $\mathrm{F}=424.3, P<0.005)$, sampling frequency $(\mathrm{F}=447.7, P<0.005)$ and Method $\mathrm{x}$ sampling frequency interaction $(\mathrm{F}=142.2, P<0.005)$. The $\mathrm{MCC}$ values increased in all methods as the sampling interval decreased, and were equal to 1 in all four methods at a sampling interval of $1 \mathrm{~h}$. LS did not function as a classifier at sampling intervals of 3-4 h. The MCC values of MICOP were 0.7 or more under all conditions.

We compared the accuracy of MICOP and existing methods for one-cycle data (Fig. 4). Among all conditions (method, noise, and sampling frequency), determination accuracies using one-cycle were lower than those using two cycles. All methods did not work under all conditions at the 4-h sampling frequency. Meanwhile, MICOP and JTK showed high performances under sampling conditions $\leq 3 \mathrm{~h}$.

\section{Reanalysis of previously reported time-resolved proteomics datasets}

We reanalyzed the time-series proteome data for mouse liver reported by Mauvoisin et al. using C57BL/6 and 


\section{a Typical decaying oscillation}
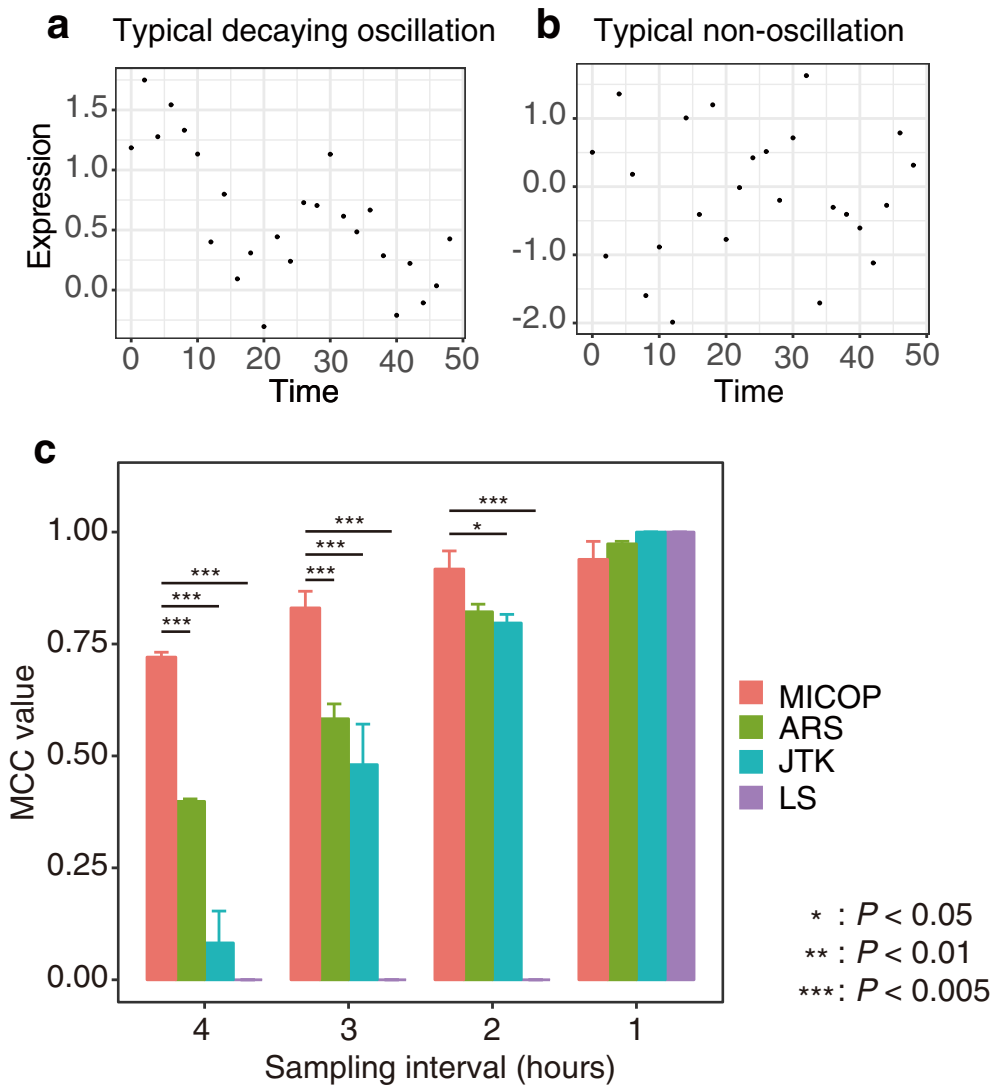

Fig. 2 MCC values of MICOP, ARS, JTK, and LS for decaying data. Comparison of detection power of MICOP and existing methods for decaying data. a Typical decaying oscillation data, $\mathbf{b}$ typical non-oscillation data, $\mathbf{c}$ MCC values from simulated time-resolved data in which half represent oscillating data, whereas the other half represent random numerical data, of which half do not oscillate. Noise level was 0.4 (standard deviation). The $x$-axis represents the MCC value, while the $y$-axis represents the sampling interval (hours). The color indicates each method: red, MICOP; green, ARS; blue, JTK; and purple, LS

those reported by Robles et al. using C57BL/6 J, as well as simulated data released by Wu et al. (Fig. 5, Table 1, Table 2) $[8,9,23]$. The numbers of significantly oscillating proteins assessed by standard harmonic regression were 9 (the $F$ test for multilinear regression, $P<0.01$ ), 9 (Fisher's exact test, $P<0.01)$, and $3(P<0.01)$ for biological data in the original work. Meanwhile, 32, 22, and 5 proteins were judged as being significantly oscillating for C57BL/6 J, C57BL/6, and Wu's simulated data by MICOP, respectively $(P<0.05)$. The numbers of proteins judged to be significantly oscillating in both the original work and MICOP were 2, 8 , and 2 for biological data, respectively. The numbers of proteins judged as being significantly oscillating for the three above-mentioned tests only by MICOP were 30, 14, and 3 for biological data, respectively.

\section{Discussion}

Although many algorithms have been developed to extract molecules with rhythmic oscillation in their levels from large-scale time-series data derived from mass spectrometry systems or DNA sequencers, it is known that the accuracy and sensitivity of such methods depend on noise, sampling frequency, and waveform. In particular, the discussion of the prediction power in conditions of decaying oscillation was insufficient. In this research, we provide MICOP, which is classified as a time-domain method, and demonstrate that the algorithm is particularly effective for detecting decaying oscillation.

We compared the detection power of MICOP and previously reported algorithms for decaying oscillation. We revealed that, in terms of the power for detection decaying oscillation, MICOP outperformed other algorithms (Fig. 2). In particular, MICOP showed a clear advantage when the sampling frequency was low. This is because MIC can effectively detect non-linear associations like associations between decaying oscillation and the reference sin curve (Fig. 1). Although we compared the performance for only cosine wave, additional experiment with peak wave or complex wave is also important. ARS showed high performance following MICOP because de-trending at preprocessing seemed to cancel out 

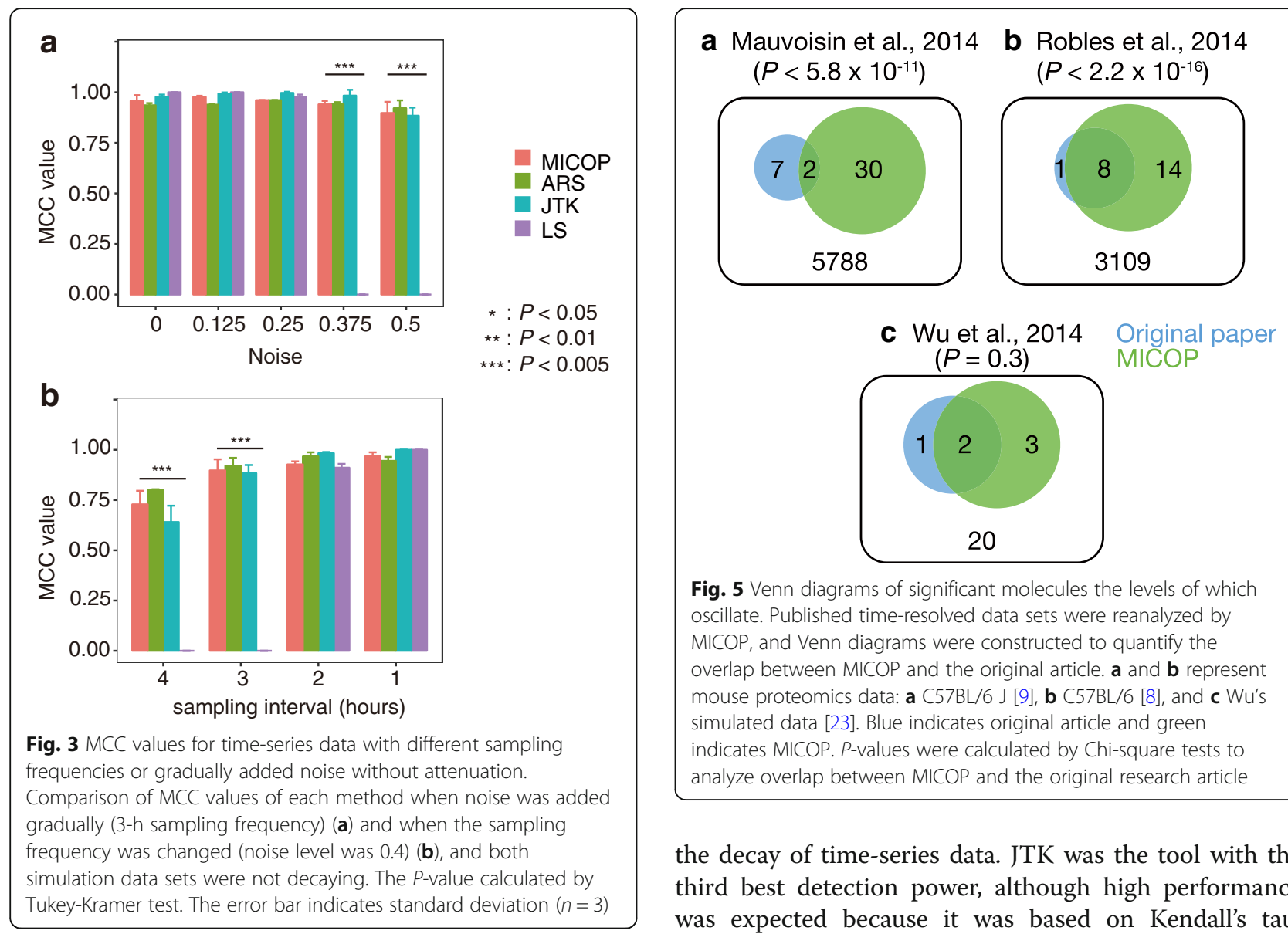

Fig. 5 Venn diagrams of significant molecules the levels of which oscillate. Published time-resolved data sets were reanalyzed by MICOP, and Venn diagrams were constructed to quantify the overlap between MICOP and the original article. $\mathbf{a}$ and $\mathbf{b}$ represent mouse proteomics data: a C57BL/6 J [9], b C57BL/6 [8], and c Wu's simulated data [23]. Blue indicates original article and green indicates MICOP. P-values were calculated by Chi-square tests to analyze overlap between MICOP and the original research article

the decay of time-series data. JTK was the tool with the third best detection power, although high performance was expected because it was based on Kendall's tau, which is a measure of rank correlation, and it did not depend on amplitude. This indicates that MICOP has

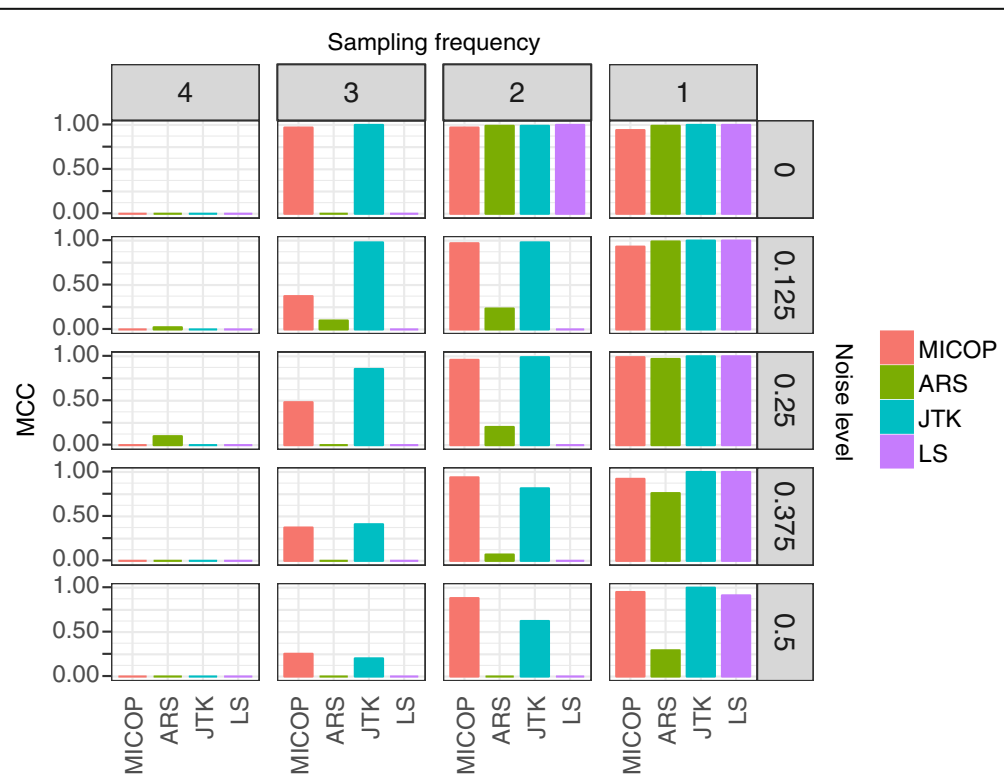

Fig. 4 MCC values for time-series data with one-cycle data. Comparison of MCC values for each method with one-cycle time-series data. Row order indicates noise level and column order indicates sampling interval (h). Colors refer to each method. Sampling frequency and noise level were gradually adjusted 
Table 1 Novel oscillating protein candidates of C57BL/6 J [9] detected by MICOP

\begin{tabular}{|c|c|c|c|c|}
\hline Gene name & Species & Condition & Tissue & Reference \\
\hline$\overline{A c o t 8}$ & - & - & - & - \\
\hline \multirow[t]{2}{*}{ Acox 1} & Homo sapiens & $\mathrm{LL}$ & blood & {$[36]$} \\
\hline & Mus musculus & LD & liver/SCN & {$[37,38]$} \\
\hline \multirow[t]{2}{*}{ Acs 15} & Mus musculus & $\mathrm{DD} / \mathrm{LD}$ & liver/SCN & [39] \\
\hline & Mus musculus & LD & $\mathrm{SCN}$ & {$[37]$} \\
\hline \multirow[t]{2}{*}{ Akr1c14 } & Mus musculus & LD & liver & {$[40]$} \\
\hline & Mus musculus & $\mathrm{DD}$ & cartilage tissue & [41] \\
\hline \multirow[t]{3}{*}{ Cbs } & Mus musculus & $\mathrm{DD} / \mathrm{LD}$ & liver & {$[42-45]$} \\
\hline & Mus musculus & LD & $\mathrm{SCN}$ & {$[37]$} \\
\hline & Homo sapiens & $\mathrm{LL}$ & blood & {$[36]$} \\
\hline Cct8 & Mus musculus & LD & $\mathrm{SCN}$ & {$[37]$} \\
\hline Cesib & - & - & - & - \\
\hline Chid 1 & Mus musculus & $\mathrm{DD} / \mathrm{LD}$ & liver/SCN & {$[46]$} \\
\hline Cxadr & Mus musculus & $\mathrm{DD} / \mathrm{LD}$ & liver/SCN & {$[42]$} \\
\hline Cyp4f14 & - & - & - & - \\
\hline \multirow[t]{2}{*}{ Gns } & Homo sapiens & $\mathrm{LL}$ & blood & {$[36]$} \\
\hline & Mus musculus & $\mathrm{DD} / \mathrm{LD}$ & liver & {$[40]$} \\
\hline Golgb 1 & Mus musculus & $\mathrm{DD} / \mathrm{LD}$ & liver/SCN & {$[37,40,47]$} \\
\hline Gpx3 & - & - & - & - \\
\hline Hars & Mus musculus & LD & liver & {$[40]$} \\
\hline $\mathrm{Hrg}$ & - & - & - & - \\
\hline Mfap4 & - & - & - & - \\
\hline Mug1 & Mus musculus & DD & liver & {$[42]$} \\
\hline Pdcd6 & Mus musculus & LD & liver/SCN & {$[37,40,47]$} \\
\hline Ptms & Mus musculus & LD & $\mathrm{SCN}$ & {$[37]$} \\
\hline Safb & - & - & - & - \\
\hline Serpina6 & Mus musculus & $\mathrm{DD} / \mathrm{LD}$ & liver & [44] \\
\hline sf3b2 & Mus musculus & LD & telogen epidermis & {$[46]$} \\
\hline Slc9a3r1 & Mus musculus & $\mathrm{DD} / \mathrm{LD}$ & liver & {$[46]$} \\
\hline Snrpd3 & Mus musculus & LD & liver & {$[47]$} \\
\hline \multirow[t]{2}{*}{ Stk38 } & Mus musculus & $\mathrm{DD}$ & liver & {$[46]$} \\
\hline & Mus musculus & LD & $\mathrm{SCN}$ & {$[37]$} \\
\hline$T p r$ & Mus musculus & $\mathrm{DD} / \mathrm{LD}$ & liver & {$[40,46]$} \\
\hline \multirow[t]{2}{*}{ Txndc15 } & Mus musculus & $\mathrm{DD} / \mathrm{LD}$ & liver & {$[46,47]$} \\
\hline & Mus musculus & LD & $\mathrm{SCN}$ & {$[37]$} \\
\hline \multirow[t]{2}{*}{ Ubl4a } & Mus musculus & LD & $\mathrm{SCN}$ & {$[37]$} \\
\hline & Mus musculus & $\mathrm{DD}$ & liver & {$[46]$} \\
\hline Uox & Mus musculus & $\mathrm{DD} / \mathrm{LD}$ & liver & {$[39,40,42,47]$} \\
\hline Ythdf2 & Mus musculus & LD & liver & {$[47]$} \\
\hline
\end{tabular}

Novel oscillating protein candidates identified by MICOP from time-series proteomics data of C57BL/6 J [9] and a list of previous papers that have experimentally demonstrated that gene expression oscillates in transcriptome analysis. LD stands for the daily 24-h light-dark (LD) cycle and DD stands for constant darkness conditions. Hyphens indicate that we could not find previous consistent works which prove the mRNA oscillation 
Table 2 Novel oscillating protein candidates of C57BL/6 [8] detected by MICOP

\begin{tabular}{|c|c|c|c|c|}
\hline Gene name & Species & Condition & Tissue & Reference \\
\hline Anp32e & Mus musculus & $\mathrm{DD} / \mathrm{LD}$ & liver & {$[39,40]$} \\
\hline Anpep & - & - & - & - \\
\hline Cgn & Mus musculus & LD & liver & {$[47]$} \\
\hline \multirow[t]{2}{*}{ Csde1 } & Mus musculus & DD & liver & [39] \\
\hline & Mus musculus & LD & $\mathrm{SCN}$ & {$[37]$} \\
\hline Enpp4 & Mus musculus & LD & liver/anagen epidermis & {$[40,46,47]$} \\
\hline \multirow[t]{2}{*}{ Gn/2 } & Mus musculus & $\mathrm{DD}$ & hippocampus/liver & {$[39,48]$} \\
\hline & Mus musculus & LD & $\mathrm{SCN}$ & {$[37]$} \\
\hline \multirow[t]{2}{*}{ Ldhb } & Homo sapiens & $\mathrm{LL}$ & blood & {$[36]$} \\
\hline & Mus musculus & LD & anagen epidermis, SCN & {$[37,46]$} \\
\hline \multirow[t]{2}{*}{ Numal } & Mus musculus & LD & liver & {$[40]$} \\
\hline & Mus musculus & DD & cartilage tissue & {$[41]$} \\
\hline $\operatorname{Prd} \times 2$ & Mus musculus & LD & $\mathrm{SCN}$ & {$[37]$} \\
\hline Rnf114 & - & - & - & - \\
\hline Slc4al & - & - & - & - \\
\hline Slcolb2 & Mus musculus & $\mathrm{DD} / \mathrm{LD}$ & liver & {$[39,40,42]$} \\
\hline Tomm70a & - & - & - & - \\
\hline Vps26a & - & - & - & - \\
\hline
\end{tabular}

excellent performance for decaying oscillation, and suggests that an MIC-based approach that can detect non-linear associations is useful to detect decaying oscillation.

Moreover, we compared the MCC values for all methods on data containing gradual Gaussian noise to test the noise resistance (Fig. 3a). As a result, MICOP showed equal performance to JTK and ARS in the range of standard deviation of $0.125-0.500$. This indicated that the performance of MICOP for noisy data is equal to that of the existing methods. This result suggests that the robustness to noise of MICOP is the same as that of well-known ARS and JTK, while the high performance of LS was limited to conditions with a low noise level. This numerical experiment revealed that the noise resistance of MICOP is the same as that of other widely used methods.

Clarifying the relationship between accuracy and sampling frequency in analyzing omics data, for which increasing the number of sampling points seems difficult, is important for determining the experimental design. As expected, with increase in the sampling frequency, the MCC values tended to increase (Figs. 2 and 3b). The fact that the ARS, JTK, and LS could characterize oscillation and non-oscillation in almost all cases when the sampling interval was $2 \mathrm{~h}$ or less is similar to the findings in original research studies of various methods and research comparing them $[11,28]$. This suggested that a high sampling frequency improved accuracy; therefore, sampling frequency should be as high as experimental constraints allow.

We applied MICOP and existing methods for one-cycle of data (Fig. 4). As expected, accuracy decreased for all methods when one-cycle was used. However, MICOP and JTK showed high MCC values among methods under this condition. Also, MICOP seems to outperformed JTK under limited conditions which is low sampling frequency and high noise for one-cycle data (Fig. 4). Human omics data often have lower sampling frequencies, high noise levels, and only one-cycle. Our results suggest that MICOP and JTK have considerable potential for analyzing human omics datasets.

We reanalyzed the time-series proteomics data of C57BL/6 J and C57BL/6 to test the performance of MICOP and explore additional candidates of proteins with rhythmic change in their expression profile $[8,9]$. These datasets include the mouse liver proteome data obtained by sampling every $3 \mathrm{~h}$ for 2 days, for which the analysis of the peptides was performed with a mass spectrometer. Approximately, 3000 protein types were detected in each study. Proteins that were detected in both MICOP and the original studies numbered 2 and 8 for C57BL/6 J and C57BL/6, respectively (Fig. 5). This actual application for proteomics data suggests that MICOP can obtain results in a manner approximately similar to 
the existing methods. Specifically, the MICOP results were consistent with those in the original articles regarding these commonly identified proteins. Furthermore, the proteins that were uniquely identified with MICOP were numbered 30 and 14 for C57BL/6 J and C57BL/6, respectively (Table 1 , Table 2). These results strongly suggest that MICOP is a powerful tool to detect proteins with rhythmic changes in their expression levels from time-resolved proteomics data.

Although mass spectrometry-based approaches have been used for proteome-level studies of circadian rhythms, completely measuring mouse proteomes remains difficult. A comprehensive transcriptome analysis with parallel sequencers has revealed that $\sim 15-20 \%$ of mouse liver mRNA significantly oscillates [29]. However, in these proteome studies of $\mathrm{C} 57 \mathrm{BL} / 6$ and $\mathrm{C} 57 \mathrm{BL} / 6 \mathrm{~J}$, significantly oscillating protein are rare $(<1 \%$ of detected total proteins; FDR $<0.05$ ), a result inconsistent with those of mouse proteome studies. Multiple factors can explain this pattern. Typical clock protein known as principle oscillators such as CRY1, CRY2, PER2, REV-ERB $\alpha$ and CLOCK have comparatively low expression levels and are not detected in these studies [8, 9]. In addition, non-Gaussian experimental noise which is specific to MS measurement hampers the application of statistical test on proteins [30]. These problems may be improved by analyzing higher quality proteome datasets with modern technologies [31, 32]. Some core circadian proteins such as CRY1, CRY2, PER2, REV-ERVa and CLOCK could be detected in recently published proteome datasets [31, 32]. Thus, the development of proteome analysis technology may resolve discrepancies between results of transcriptome analysis and proteome analysis, and clarify connections within the circadian rhythm transcription and translation network.

We present a new list of proteins that oscillate by MICOP (Tables 1 and 2). The accuracy of these estimates is difficult to ascertain. Interestingly, when examining expression patterns of genes encoding these proteins, we estimated that the proteins were new oscillating molecules in MICOP. In addition, a large fraction of candidates was presumed to oscillate in a previous transcriptome analysis [29]. Two independent studies which measured both transcriptome and proteome of human samples revealed that only $30 \%$ of mRNA-protein correlation had statistically significant [33, 34]. This fact suggested that even if mRNA abundance is oscillating, protein abundance may not be always oscillating. However, about $90 \%$ of mRNA-protein correlation showed positive, hence rhythmic mRNA expression suggests the possibility of protein oscillation [34]. An overlap between re-analyzed proteomics data by MICOP and transcriptome analysis showed a consistent result.
MICOP accuracy tends to be low for data that do not perfectly fit a sine curve. The periodicity that MICOP can detect is subject to the shape of the reference curve, so changing the reference curve is necessary to detect asymmetric waveforms including saw tooth-like shapes like RAIN [30]. Furthermore, adjusting the false discovery rate is essential for accurate prediction, since MICOP repeats the hypothesis tests. In addition, verification with additional data such as periodic peak wave or overlapping sine wave is necessary in order to evaluate the accuracy of MICOP more precisely. Judgments of phase and cycle are possible in principle, but we did not perform them; therefore, this should be considered in future studies. Mutual information increased when sample size was small and correlation between two variables was null, even when the variables were random [35]. We solved this issue in MICOP by determining the $P$-value with the Monte Carlo method. When the time points (sample size) are small, the criterion for calculating the $P$-value increases, and when the time points are large, the criterion for calculating the $P$-value decreases (Additional file 3 ). In this paper, we presented MICOP, which is an MIC-based algorithm, for predicting periodic patterns in large-scale time-resolved protein expression profiles. The performance test using artificially generated simulation data revealed that the performance of MICOP for decaying data was superior to that of the existing widely used methods. Additionally, we indicated that MICOP is compatible with noisy data obtained with a low sampling frequency. Furthermore, the performance test using actual mouse proteomics data suggested that MICOP may be able to provide novel findings from proteomics data. Specifically, it can reveal novel findings from time-series data and may contribute to biologically significant results. This study suggests that MICOP is an ideal approach for detecting and characterizing oscillations in time-resolved omics data sets.

\section{Conclusion}

In this paper, we presented MICOP, which is an MIC-based algorithm, for predicting periodic patterns in large-scale time-resolved protein expression profiles. The performance test using artificially generated simulation data revealed that the performance of MICOP for decaying data was superior to that of the existing widely used methods. Additionally, we indicated that MICOP is compatible with noisy data obtained with a low sampling frequency. Furthermore, the performance test using actual mouse proteomics data suggested that MICOP may be able to provide novel findings from proteomics data. Specifically, it can reveal novel findings from time-series data and may contribute to biologically significant results. This study suggests that MICOP is an ideal approach for detecting and characterizing oscillations in time-resolved omics data sets. 


\section{Additional files}

Additional file 1: Wide range comparison of MCC values of MICOP, ARS, JTK, and LS for decaying data. Sampling interval and noise level were gradually adjusted. The bar indicates MCC values (1 indicates a perfect prediction, 0 indicates a random prediction, and -1 indicates a prediction in complete disagreement). (PDF 75 kb)

Additional file 2: Wide-range comparison of MCC values of MICOP, ARS, JTK, and LS for non-decaying data. Sampling interval and noise level were gradually adjusted. The bar indicates MCC values (1 indicates a perfect prediction, 0 indicates a random prediction, and -1 indicates a prediction in complete disagreement). (PDF $75 \mathrm{~kb}$ )

Additional file 3: Monte-Carlo simulation to calculate $P$-values. MIC values were calculated between random numbers. The $\mathrm{x}$-axis indicates sample number ( $\mathrm{N}$ time points) and the $\mathrm{y}$-axis indicates MIC. The error bar indicates the standard deviation $(N=1000)$. The red color represents random values and the blue color represents the significance threshold (5\%). (PDF $68 \mathrm{~kb}$ )

\section{Abbreviations}

ARS: Autoregressive spectral estimation; FN: False Negative; FP: False positive; JTK: Jonckheere-Terpstra (JT) test and obtained the strength of correlation by Kendal's tau test; LS: Lomb-Scargle; MCC: Mathews correlation coefficient; MIC: Maximal information coefficient; MICOP: Maximal information coefficient-based oscillation prediction; MINE: Maximal information-based nonparametric estimation; TN: True negative; TP: True positive

\section{Funding}

This work was supported by research funds from the Yamagata Prefectural Government and by research funds from Tsuruoka City, Japan.

\section{Availability of data and materials}

The scripts for analysis were uploaded on the following URL. https:// docs.google.com/document/d/

1bN44qAJFP9O6BTTA_0ameil9py0LS3rcXKT2cxbKTwY/edit?usp=sharing

\section{Authors' contributions}

$\mathrm{HI}$ conducted the bioinformatics analyses. MS supervised the project. $\mathrm{HI}$ and MS wrote the manuscript. MT supported the writing of the manuscript. All authors have read and approved the final manuscript.

\section{Ethics approval and consent to participate}

Not applicable.

\section{Consent for publication}

Not applicable.

\section{Competing interests}

The authors declare that they have no competing interests.

\section{Publisher's Note}

Springer Nature remains neutral with regard to jurisdictional claims in published maps and institutional affiliations.

\footnotetext{
Author details

${ }^{1}$ Systems Biology Program, Graduate School of Media and Governance, Keio University, Fujisawa 252-8520, Japan. ${ }^{2}$ Institute for Advanced Biosciences, Keio University, Tsuruoka 997-0052, Japan. ${ }^{3}$ Health Promotion and Preemptive Medicine, Research and Development Center for Minimally Invasive Therapies, Tokyo Medical University, Shinjuku, Tokyo 160-0022, Japan. ${ }^{4}$ Department of Environment and Information Studies, Keio University, Fujisawa 252-8520, Japan.
}

Received: 12 October 2017 Accepted: 20 June 2018 Published online: 28 June 2018

\section{References}

1. Mohawk JA, Green CB, Takahashi JS. Central and peripheral circadian clocks in mammals. Annu Rev Neurosci. 2012;35:445-62.
2. Koike N, Yoo SH, Huang HC, Kumar V, Lee C, Kim TK, Takahashi JS Transcriptional architecture and chromatin landscape of the core circadian clock in mammals. Science. 2012;338(6105):349-54.

3. Partch $\mathrm{CL}$, Green $\mathrm{CB}$, Takahashi JS. Molecular architecture of the mammalian circadian clock. Trends Cell Biol. 2014;24(2):90-9.

4. Weitzman ED, Fukushima D, Nogeire C, Roffwarg H, Gallagher TF, Hellman L. Twenty-four hour pattern of the episodic secretion of cortisol in normal subjects. J Clin Endocrinol Metab. 1971;33(1):14-22.

5. Kennaway DJ, Voultsios A, Varcoe TJ, Moyer RW. Melatonin in mice: rhythms, response to light, adrenergic stimulation, and metabolism. Am J Physiol Regul Integr Comp Physiol. 2002;282(2):R358-65.

6. Kasukawa T, Sugimoto M, Hida A, Minami Y, Mori M, Honma S, Honma K, Mishima K, Soga T, Ueda HR. Human blood metabolite timetable indicates internal body time. Proc Natl Acad Sci U S A. 2012;109(37):15036-41.

7. Minami Y, Kasukawa T, Kakazu Y, ligo M, Sugimoto M, Ikeda S, Yasui A, van der Horst GT, Soga T, Ueda HR. Measurement of internal body time by blood metabolomics. Proc Natl Acad Sci U S A. 2009:106(24):9890-5.

8. Robles MS, Cox J, Mann M. In-vivo quantitative proteomics reveals a key contribution of post-transcriptional mechanisms to the circadian regulation of liver metabolism. PLoS Genet. 2014;10(1):e1004047.

9. Mauvoisin D, Wang J, Jouffe C, Martin E, Atger F, Waridel P, Quadroni M, Gachon F, Naef F. Circadian clock-dependent and -independent rhythmic proteomes implement distinct diurnal functions in mouse liver. Proc Natl Acad Sci U S A. 2014;111(1):167-72.

10. Ono D, Honma K, Honma S. Circadian and ultradian rhythms of clock gene expression in the suprachiasmatic nucleus of freely moving mice. Sci Rep. 2015:5:12310.

11. Deckard A, Anafi RC, Hogenesch JB, Haase SB, Harer J. Design and analysis of large-scale biological rhythm studies: a comparison of algorithms for detecting periodic signals in biological data. Bioinformatics. 2013;29(24): 3174-80.

12. Agostinelli F, Ceglia N, Shahbaba B, Sassone-Corsi P, Baldi P. What time is it? Deep learning approaches for circadian rhythms. Bioinformatics. 2016;32(12): i8-i17.

13. Ukai-Tadenuma M, Yamada RG, Xu H, Ripperger JA, Liu AC, Ueda HR. Delay in feedback repression by cryptochrome 1 is required for circadian clock function. Cell. 2011;144(2):268-81.

14. Chudova D, Ihler A, Lin KK, Andersen B, Smyth P. Bayesian detection of nonsinusoidal periodic patterns in circadian expression data. Bioinformatics. 2009;25(23):3114-20.

15. Harmer SL, Hogenesch JB, Straume M, Chang HS, Han B, Zhu T, Wang X, Kreps JA, Kay SA. Orchestrated transcription of key pathways in Arabidopsis by the circadian clock. Science. 2000;290(5499):2110-3.

16. Straume M. DNA microarray time series analysis: automated statistical assessment of circadian rhythms in gene expression patterning. Methods Enzymol. 2004;383:149-66.

17. Hughes ME, Hogenesch JB, Kornacker K. JTK_CYCLE: an efficient nonparametric algorithm for detecting rhythmic components in genomescale data sets. J Biol Rhythm. 2010;25(5):372-80.

18. Wichert S, Fokianos K, Strimmer K. Identifying periodically expressed transcripts in microarray time series data. Bioinformatics. 2004;20(1):5-20.

19. Takalo $\mathrm{R}$, Hytti $\mathrm{H}$, Ihalainen $\mathrm{H}$. Tutorial on univariate autoregressive spectral analysis. J Clin Monit Comput. 2005;19(6):401-10.

20. Yang R, Su Z. Analyzing circadian expression data by harmonic regression based on autoregressive spectral estimation. Bioinformatics. 2010;26(12): i168-74.

21. Levine JD, Funes P, Dowse HB, Hall JC. Signal analysis of behavioral and molecular cycles. BMC Neurosci. 2002;3:1.

22. Langmead CJ, Yan AK, McClung CR, Donald BR. Phase-independent rhythmic analysis of genome-wide expression patterns. J Comput Biol. 2003, 10(3-4):521-36.

23. Wu G, Zhu J, Yu J, Zhou L, Huang JZ, Zhang Z. Evaluation of five methods for genome-wide circadian gene identification. J Biol Rhythm. 2014;29(4):231-42

24. Wu G, Anafi RC, Hughes ME, Kornacker K, Hogenesch JB. MetaCycle: an integrated $R$ package to evaluate periodicity in large scale data. Bioinformatics. 2016:32(21):3351-3.

25. Matthews BW. Comparison of the predicted and observed secondary structure of T4 phage lysozyme. Biochim Biophys Acta. 1975:405(2):442-51.

26. Wu G, Anafi RC, Hughes ME, Kornacker K, Hogenesch JB. MetaCycle: an integrated $\mathrm{R}$ package to evaluate periodicity in large scale data. Bioinformatics. 2016;32(21):3351-53. 
27. Team RC. R: A Language and Environment for Statistical Computing. Vienna, Austria: R Foundation for Statistical Computing; 2017

28. Cohen GB, Rangan VS, Chen BK, Smith S, Baltimore D. The human thioesterase II protein binds to a site on HIV-1 Nef critical for CD4 downregulation. J Biol Chem. 2000;275(30):23097-105.

29. Zhang R, Lahens NF, Ballance HI, Hughes ME, Hogenesch JB. A circadian gene expression atlas in mammals: implications for biology and medicine. Proc Natl Acad Sci U S A. 2014;111(45):16219-24.

30. Thaben PF, Westermark PO. Detecting rhythms in time series with RAIN. J Biol Rhythm. 2014;29(6):391-400.

31. Robles MS, Humphrey SJ, Mann M. Phosphorylation is a central mechanism for circadian control of metabolism and physiology. Cell Metab. 2017;25(1): 118-27.

32. Wang J, Mauvoisin D, Martin E, Atger F, Galindo AN, Dayon L, Sizzano F, Palini A, Kussmann M, Waridel P, et al. Nuclear proteomics uncovers diurnal regulatory landscapes in mouse liver. Cell Metab. 2017;25(1):102-17.

33. Gry M, Rimini R, Stromberg S, Asplund A, Ponten F, Uhlen M, Nilsson P. Correlations between RNA and protein expression profiles in 23 human cell lines. BMC Genomics. 2009;10:365.

34. Zhang B, Wang J, Wang X, Zhu J, Liu Q, Shi Z, Chambers MC, Zimmerman $\sqcup$, Shaddox KF, Kim S, et al. Proteogenomic characterization of human colon and rectal cancer. Nature. 2014;513(7518):382-7.

35. Steuer R, Kurths J, Daub CO, Weise J, Selbig J. The mutual information: detecting and evaluating dependencies between variables. Bioinformatics. 2002;18(Suppl 2):S231-40.

36. Moller-Levet CS, Archer SN, Bucca G, Laing EE, Slak A, Kabiljo R, Lo JC, Santhi $\mathrm{N}$, von Schantz $\mathrm{M}$, Smith $\mathrm{CP}$, et al. Effects of insufficient sleep on circadian rhythmicity and expression amplitude of the human blood transcriptome. Proc Natl Acad Sci U S A. 2013;110(12):E1132-41.

37. Pembroke WG, Babbs A, Davies KE, Ponting CP, Oliver PL. Temporal transcriptomics suggest that twin-peaking genes reset the clock. Elife. 2015; 4. https://www.ncbi.nlm.nih.gov/pubmed/26523393.

38. Goh BC, Wu X, Evans AE, Johnson ML, Hill MR, Gimble JM. Food entrainment of circadian gene expression altered in PPARalpha-/- brown fat and heart. Biochem Biophys Res Commun. 2007;360(4):828-33.

39. Hughes ME, DiTacchio L, Hayes KR, Vollmers C, Pulivarthy S, Baggs JE, Panda $\mathrm{S}$, Hogenesch JB. Harmonics of circadian gene transcription in mammals. PLoS Genet. 2009;5(4):e1000442

40. Barclay JL, Husse J, Bode B, Naujokat N, Meyer-Kovac J, Schmid SM, Lehnert $\mathrm{H}$, Oster $\mathrm{H}$. Circadian desynchrony promotes metabolic disruption in a mouse model of shiftwork. PLoS One. 2012;7(5):e37150.

41. Gossan N, Zeef L, Hensman J, Hughes A, Bateman JF, Rowley L, Little CB, Piggins HD, Rattray M, Boot-Handford RP, et al. The circadian clock in murine chondrocytes regulates genes controlling key aspects of cartilage homeostasis. Arthritis Rheum. 2013;65(9):2334-45.

42. Miller BH, McDearmon EL, Panda S, Hayes KR, Zhang J, Andrews JL, Antoch MP, Walker JR, Esser KA, Hogenesch JB, et al. Circadian and CLOCKcontrolled regulation of the mouse transcriptome and cell proliferation. Proc Natl Acad Sci U S A. 2007:104(9):3342-7.

43. Tsuchiya H, da Costa KA, Lee S, Renga B, Jaeschke H, Yang Z, Orena SJ, Goedken MJ, Zhang Y, Kong B, et al. Interactions between nuclear receptor SHP and FOXA1 maintain oscillatory homocysteine homeostasis in mice. Gastroenterology. 2015;148(5):1012-23. e1014

44. Janich P, Arpat AB, Castelo-Szekely V, Lopes M, Gatfield D. Ribosome profiling reveals the rhythmic liver translatome and circadian clock regulation by upstream open reading frames. Genome Res. 2015;25(12): 1848-59.

45. Vollmers C, Schmitz RJ, Nathanson J, Yeo G, Ecker JR, Panda S. Circadian oscillations of protein-coding and regulatory RNAs in a highly dynamic mammalian liver epigenome. Cell Metab. 2012;16(6):833-45.

46. Geyfman M, Kumar V, Liu Q, Ruiz R, Gordon W, Espitia F, Cam E, Millar SE, Smyth P, Ihler A, et al. Brain and muscle Arnt-like protein-1 (BMAL1) controls circadian cell proliferation and susceptibility to UVB-induced DNA damage in the epidermis. Proc Natl Acad Sci U S A. 2012;109(29):11758-63.

47. Eckel-Mahan KL, Patel VR, de Mateo S, Orozco-Solis R, Ceglia NJ, Sahar S, Dilag-Penilla SA, Dyar KA, Baldi P, Sassone-Corsi P. Reprogramming of the circadian clock by nutritional challenge. Cell. 2013;155(7):1464-78.

48. Renaud J, Dumont F, Khelfaoui M, Foisset SR, Letourneur F, Bienvenu T, Khwaja O, Dorseuil O, Billuart P. Identification of intellectual disability genes showing circadian clock-dependent expression in the mouse hippocampus. Neuroscience. 2015;308:11-50.

\section{Ready to submit your research? Choose BMC and benefit from:}

- fast, convenient online submission

- thorough peer review by experienced researchers in your field

- rapid publication on acceptance

- support for research data, including large and complex data types

- gold Open Access which fosters wider collaboration and increased citations

- maximum visibility for your research: over $100 \mathrm{M}$ website views per year

At BMC, research is always in progress.

Learn more biomedcentral.com/submissions 